\title{
Lung Ultrasound in Pediatric Asthma - Characterizing Baseline Findings Outside of Acute Exacerbations
}

\author{
Noah Marzook $^{1}$, Francois Gagnon ${ }^{2}$, Alexandre Deragon ${ }^{3}$, David Zielinski ${ }^{1}$, Adam Shapiro ${ }^{4}$, \\ Larry Lands ${ }^{5}$, and Alexander S. Dubrovsky ${ }^{6}$ \\ ${ }^{1}$ McGill University \\ ${ }^{2}$ University of Ottawa Department of Pediatrics \\ ${ }^{3}$ McGill University Faculty of Medicine \\ ${ }^{4}$ Montreal Children's Hospital, McGill University \\ ${ }^{5}$ McGill University Health Centre \\ ${ }^{6}$ McGill Univ
}

December 16, 2021

\begin{abstract}
Background: Lung ultrasound (LUS) has been shown to be an effective tool to rapidly diagnose certain causes of pediatric respiratory distress in the emergency department. However, very little is known about LUS findings in pediatric asthma outside of acute exacerbations. Objectives: The primary objective of this study was to characterize LUS findings in a cohort of pediatric patients with a definitive diagnosis of asthma, outside of an asthma exacerbation. Methods: Eligible patients, aged 6 to 17 years old and diagnosed with asthma, underwent LUS during an outpatient visit. LUS was conducted using a six-zone scanning protocol. A positive LUS was defined by one or more of the following: [?]3 B-lines per intercostal space, pulmonary consolidation and/or pleural anomaly. Images were interpreted by an expert sonographer blinded to patient clinical characteristics. Results: 52 patients were included. 10/52 patients had a positive LUS (19.2\%, 95CI 8.3-30.1\%). Of those with positive LUS findings, 8 had B lines, 7 had consolidations $<1 \mathrm{~cm}, 1$ had a pleural line abnormality and 1 had a consolidation $>1 \mathrm{~cm}$. Positive findings were seen in the right anterior and lateral zones in $60 \%$ of participants and were limited to 1-2 intercostal spaces within one lung zone in $100 \%$ of participants. Conclusion: To our knowledge, this is the first report of LUS findings in outpatient pediatric asthma. Positive LUS findings in asthmatic children can be seen outside of acute exacerbations. Such findings need to be taken into consideration when using LUS for the acute evaluation of a pediatric patient with asthma.
\end{abstract}

\section{Introduction}

Asthma represents one of the most common chronic conditions of childhood ${ }^{1-4}$. It is a leading cause for both pediatric emergency department (PED) visits and hospital admissions amongst children. Despite all of the investigational tools available to physicians, it can sometimes be difficult to clinically discriminate acute asthma from other diagnoses, including pulmonary infection, lobar atelectasis and pneumothorax. Thus, the diagnosis of asthma often relies on clinical features found through acquiring a thorough medical history, and conducting a physical examination ${ }^{5,6}$. Lung ultrasound (LUS) has emerged as a rapid, bedside method of investigating respiratory distress ${ }^{7}$. It has proven to be both sensitive and specific in diagnosing common pediatric respiratory pathologies such as pneumonia, pneumothorax, pulmonary effusion and neonatal lung diseases $^{8-13}$. Lung ultrasound has also been compared to extent of parenchymal changes on CT scans in the pediatric population and to extent of lung disease in different pathologies for which lung ultrasound scoring systems have been developed ${ }^{12,14-18}$.

Previous studies have looked at LUS in pediatric patients with asthma when presenting to the PED in 
respiratory distress ${ }^{19,20}$. A study by our group found that $45 \%$ of children presenting with an acute asthma exacerbation had a positive LUS ${ }^{20}$. The cause of these findings is uncertain. It was hypothesized these findings were related to possible concomitant respiratory infections, since viral upper respiratory tract infection is the most common cause of asthma exacerbation in pediatric patients ${ }^{21}$. However, a more in-depth understanding of the LUS findings in children with asthma is required for its routine application in the acute PED setting.

The primary objective of this study was to characterize LUS findings in a cohort of pediatric patients with a definitive diagnosis of asthma (confirmed by spirometry or methacholine challenge test (MCT)) who were not experiencing an asthma exacerbation and were at their baseline state of health. The secondary objective was to compare the clinical characteristics of participants with positive LUS to those with negative LUS, in order to identify potential predictors of abnormal LUS findings.

\section{Method}

This prospective, cross-sectional study was conducted from December 2017 to June 2019 in the pediatric respiratory clinic of the McGill University Health Centre. Approval for this study was granted by the hospital's Institutional Research Ethics Board (Study 2018-3907). Eligible patients were prospectively recruited in the pediatric respirology clinic when a study sonographer was available. Written informed assent and consent were obtained from participants and their guardians.

The sample size was calculated on the basis of the previous study of children presenting to the emergency room for an acute asthma exacerbation, where approximately $20 \%$ of the patients above 6 years of age had a positive lung ultrasound ${ }^{20}$. Assuming a similar rate of lung ultrasound findings in our cohort and defining a precision of $\pm 10 \%$ as clinically meaningful, a sample size of 52 patients would provide $90 \%$ confidence that the true rate is within these precision limits.

Patients were approached if they were 6 to 17 years old, and either known asthmatics presenting for a routine visit with spirometry or suspected asthmatics presenting for confirmatory methacholine challenge test (MCT) in the pulmonary function lab. Patients were recruited if they had a diagnosis of asthma with a positive spirometry or MCT result consistent with a diagnosis of asthma. The reference values for spirometry were obtained from the global lung function initiative ${ }^{22}$. A positive spirometry result was defined as an $\mathrm{FEV}_{1}<80 \%$ predicted as well as an $\mathrm{FEV}_{1} / \mathrm{FVC}<85$ and $12 \%$ increase in $\mathrm{FEV}_{1}$ after administration of a bronchodilator. A positive MCT result was defined as PC20 less than $8 \mathrm{mg} / \mathrm{ml}$, where PC20 represents the provocative concentration that resulted in a $20 \%$ decrease in $\mathrm{FEV}_{1}{ }^{17,23}$. We excluded patients with history of viral illness in the previous 4 weeks. Eligibility was determined through a combination of administered questionnaires and review of the electronic medical record. At enrollment, all participants and parents completed a pre-test questionnaire (Appendix A) to record overall respiratory health and medication compliance, the ISAAC Asthma Core Questionnaire, and the Asthma Control Questionnaire.

Before the start of the study, all sonographers (AD, NM, FG) received formal LUS training from an expert sonographer (ASD). An introductory course on LUS was given by the latter followed by 5 proctored ultrasounds. The principal investigator (ASD) is certified as an independent practitioner by the Canadian Emergency Ultrasound Society and has more than 10 years of experience with point of care ultrasound.

Using the z.one ultra ultrasound and the L14-3 linear transducer (Mindray, Zonare Medical System, Mahwa, NJ), each LUS was completed by one of three study sonographers. A six-zone scanning protocol was performed, comparable to that described by Copetti and Cattarossi ${ }^{24}$. Settings included a depth of $6-8 \mathrm{~cm}$ at a fundamental frequency (i.e., tissue harmonic imaging turned off) of $12 \mathrm{MHz}$. Ultrasound gel was layered on the probe and placed in a longitudinal manner over 6 zones (right and left anterior, mid-axillary and posterior chest zones). Six-second video clips were taken in each of the six zones. The images were reviewed and interpreted by the expert sonographer (ASD) who was blinded to all participant clinical information.

The LUS findings were recorded as present or absent for each of the following abnormal sonographic findings in each of the 6 lung zones, as defined by the 2012 international evidence-based recommendations for point- 
of-care ultrasound (POCUS) ${ }^{25}$ : lung slide, multiple B lines, consolidation (defined as large if [?]1 $\mathrm{cm}$ or small if $<1 \mathrm{~cm}$ ), pleural line abnormality and pleural effusion. A negative lung ultrasound was defined by the absence of any of these abnormal findings, the presence of normal lung sliding and a normal A-line pattern in each zone. Asthma control was assessed via the Asthma Control Questionnaire (ACQ). We defined asthma as being poorly controlled if the ACQ score was $>1.5^{26}$. We used the ISAAC Core Questionnaire to determine whether asthma was more likely to be severe ${ }^{27}$. The score is considered positive if patients had $>4$ attacks of wheezing, or $>1$ night per week sleep disturbance from wheeze or wheeze affecting speech in the past 12 months. This definition is based on previous ISAAC analyses showing a combination of these features correlated more closely with asthma mortality and hospital admissions ${ }^{28}$.

A post-hoc analysis was done on positive lung ultrasounds to quantify abnormalities through a scoring system that we derived derived from previous studies looking at respiratory pathology and extent of LUS findings ${ }^{14,15,29}$. This was done using the LUS quantitative scoring system, (E-Appendix B). With a score of 0-2 for each of the 6 lung zones (maximum score 12).

\section{Data analysis}

Descriptive statistics were calculated for continuous and categorical variables, as medians with interquartile ranges and frequencies, respectively. We assessed associations between LUS results and patient characteristics (see Table 1) using univariate analysis (T-test for continuous variables and Fisher's exact test for binary variables). Because some of the participants were seen at the clinic for an MCT in order to test for possible asthma, we felt they may have different characteristics compared to patients who were seen in follow up for their asthma control. Thus, we carried out a sub-analysis in both groups, those known to be asthmatic and those who came for an MCT to test for asthma.

P-values $<0.05$ were considered significant. Data were analyzed with SPSS statistics (version 23; IBM, Armonk, NY).

Results

76 patients were approached to participate in the study; 7 patients did not complete the study due to noncooperation with the LUS examination $(n=3)$ or poor LUS image quality $(n=4)$. A total of 52 participants were included in the final analysis (Figure 1). Clinical characteristics of those who completed the study are presented in Table 1 . The majority of patients were male (66\%) with a median age of 11 years (IQR 8, 14). Thirty-three (63\%) patients were diagnosed with asthma via positive MCT, and the remainder had a positive spirometry diagnoses. A large portion (42\%) of patients had asthma that was categorized as severe following the ISAAC score, and $16 \%$ had poorly controlled asthma on ACQ questionnaires.

Ten of the 52 patients had a positive LUS (19.2\%, [95CI 8.3\%-30.1\%]). Of the patients with a positive LUS, 8 had [?]3 $\mathrm{B}$ lines in an intercostal space, 7 had small subpleural consolidations $(<1 \mathrm{~cm}), 1$ had a larger consolidation $(>1 \mathrm{~cm}$ ) and 1 had a pleural line abnormality (Table 2), (Figure 2). All the findings were isolated within one to two intercostal spaces in all the participants. There was a predilection for abnormalities to be in the right side, primarily on the lateral and anterior LUS zones (6/10 positive LUS), (Figure 3).

For the 10 patients with a positive LUS, their median $\mathrm{FEV}_{1}$ score on the day of recruitment was $100 \%$ predicted, while 4 had severe asthma per the ISAAC definition. Univariate analysis was performed to look at LUS positivity and the relationship with various clinical asthma symptoms (Table 1), however there were no significant associations. LUS positivity did not show an association with asthma severity per the ISAAC criteria $(p=0.579)$ or poorly controlled asthma per ACT score $(p=0.557)$. Further analysis looking at patients with reported wheeze in the past week $(4 / 10, p=0.478)$ or recent SABA use $(8 / 10, p=0.165)$ did not show an association with LUS either.

A subanalysis was carried out in participants who were already followed at the clinic for asthma control and those who had a positive MCT result on the day of the visit for asthma diagnosis. The results are shown in Table 3. There were no significant associations found in the subanalysis between having a positive LUS in each individual group and the different variables. This analysis was only exploratory as it was not powered 
appropriately. When comparing the two groups, there is a higher rate of positive LUS in the known asthma group compared to those who presented for a confirmatory MCT (6/19 vs 4/33). Analysis to look at different variables between the two groups found that there was a significant difference in the number of patients with lower $\mathrm{FEV}_{1}$ (p 0.012), $\mathrm{FEV}_{1} / \mathrm{FVC}(\mathrm{p}<0.001)$ and the use of ICS/LABA (0.004) for maintenance therapy in the known asthmatic group compared to patients who presented for an MCT to diagnose asthma.

Using the LUS quantitative scoring system, (E-Appendix B), 9/10 of the patients had a score of 1 , and only one patient had a score of 2 (patient with a $>1 \mathrm{~cm}$ consolidation).

\section{Discussion}

This is the first study characterizing LUS findings of pediatric asthma patients at their baseline state of health. Our study showed that almost $20 \%$ of children diagnosed with asthma had positive LUS findings when well. The most frequent findings seen were small consolidations and localized B-lines. These findings may be present at baseline and are not to be attributed to, for example, a co-existing pneumonia or viral upper respiratory tract infection.

An important question to address is how our described findings may impact the evaluation of an asthmatic patient with LUS. The findings we have identified (mostly localized B-Lines in one intercostal space and small consolidations less than $1 \mathrm{~cm}$ ) are similar to what was described in previous studies, commonly seen in patients with mild bronchiolitis, atelectasis and viral pneumonia ${ }^{12,18,30}$. Therefore, using LUS to try and differentiate these conditions from baseline asthma findings is not possible.

The findings on LUS in our cohort of patients were objectively small and often localized to one lung zone, effecting no more than 2 rib spaces. LUS scores were also quite low (signifying mild changes) in most of the positive LUS cases. Thus, more severe LUS findings including consolidations $>1 \mathrm{~cm}$ and B-lines involving more than 2 intercostal spaces or effecting more than one lung zone, are atypical for a pediatric asthmatic patient at baseline and should be considered clinically significant, alerting the clinician to other possible lung parenchyma changes outside of what is typically seen in pediatric asthmatic patients.

Positive LUS findings in this study were more frequently observed on the right anterior and lateral lung zones.(Figure 3). These findings may correspond to the right middle lobe, where atelectasis is commonly seen in asthmatic patients ${ }^{31}$. We hypothesizese that these LUS findings are from sub-segmental mucus plugging and atelectasis with the known hypersecretory issues in asthma ${ }^{30,32-34}$

As a secondary objective, we explored the association of having severe or poorly controlled asthma with a positive LUS. The majority of our population had relatively mild asthma with a median of $96 \%$, and only $17 \%$ had an ACQ score that is considered elevated. There was no significant association between having positive LUS and poor asthma control, high ISAAC score, or FEV1 value. However, there was a trend toward more frequent LUS abnormalities in patients already followed in the respiratory clinic for asthma. These previously known asthmatics had significantly lower spirometry FEV1\% predicted and were more likely to be on maintenance therapy which included inhaled corticosteroid combined and long-acting beta agonist than those who presented to the clinic for asthma diagnosis with MCT (Table 3). Our LUS correlations with asthma control or severity are only exploratory and further research should be done explore these possible associations.

There are some limitations to this study. First, the sample size was relatively small (yet adequately powered), and our recruited asthmatics had overall mild disease. A larger and more severe asthma population may detect significant relationships between different clinical variables and likelihood of having a positive LUS. Second, sonographers attempted to perform LUS before spirometry maneuvers, but some known asthmatics had their scans completed after spirometry testing, which routinely included the administration of Ventolin. This was not the case for any of the MCT patients, as the scans were always done before the test. Both spirometry maneuvers and beta agonists may have altered findings on LUS. Three different sonographers performed the LUS evaluations, and ultrasound images can often be influenced by operator specific practices. However, the blinded interpretation by a single expert makes this operator bias less of an issue, and prior 
studies show good interrater reliability between sonographers and interpreters ${ }^{19,20}$. Four the ISAAC score was used in our study as a surrogate measure to asthma severity, however it is not in itself a true marker of severe asthma; the score was only used as a proxy in an attempt to explore the association of LUS findings with more severe asthma. Finally, reliance upon parental and patient questionnaires to gauge asthma severity and control may have introduced recall bias, as asthma symptoms and severity are often under-recognized by patients and parents.

\section{Conclusion}

In conclusion, up to $20 \%$ of patients with asthma may have positive LUS findings when not experiencing an acute asthma exacerbation. Most of these findings were on the right lateral or right anterior zone (60\%), mild in severity, and limited to 1-2 intercostal spaces within one lung zone. Such findings need to be taken into consideration when using LUS for the evaluation of a pediatric patient with an acute asthma exacerbation.

\section{Conflict of interests}

None.

\section{Author contribution statement}

ASD, AD, LL, AS and DZ conceived the study. ASD, NM and AD supervised the conduct of the study and data collection. AD, NM and FG performed LUS. The data, including quality control was managed by GA, FG, NM and ASD. ER provided statistical advice. NM, FG and ASD analyzed the data. NM drafted the manuscript.

\section{Acknowledgement}

The authors acknowledge Gregory Anderson, Raphael Freitas and Joanne Ramil for their help and dedication to the project, as well as Emmanouil Rampanakis for his support in statistical analysis.

\section{References}

1. Garner R, Kohen D. 2008. Changes in the prevalence of asthma among canadian children. Health Rep. 19(2):45-50.

2. Loftus PA, Wise SK. 2015. Epidemiology and economic burden of asthma. Int Forum Allergy Rhinol. 5 Suppl 1:S7-10.

3. Garner R, Kohen D. 2008. Changes in the prevalence of asthma among canadian children. Health Reports. 19(2):45.

4. . Epidemiology and economic burden of asthma. International forum of allergy \& rhinology; 2015: Wiley Online Library.

5. Ortiz-Alvarez O, Mikrogianakis A. 2012. Managing the paediatric patient with an acute asthma exacerbation. Paediatr Child Health. 17(5):251-262.

6. Hammer J. 2013. Acute respiratory failure in children. Paediatr Respir Rev. 14(2):64-69.

7. Lichtenstein DA, Meziere GA. 2008. Relevance of lung ultrasound in the diagnosis of acute respiratory failure: The blue protocol. Chest. 134(1):117-125.

8. Copetti R, Cattarossi L. 2007. The 'double lung point': An ultrasound sign diagnostic of transient tachypnea of the newborn. Neonatology. 91(3):203-209.

9. Copetti R, Cattarossi L, Macagno F, Violino M, Furlan R. 2008. Lung ultrasound in respiratory distress syndrome: A useful tool for early diagnosis. Neonatology. 94(1):52-59.

10. Copetti R, Cattarossi L. 2008. Ultrasound diagnosis of pneumonia in children. La radiologia medica. 113(2):190-198. 
11. Pereda MA, Chavez MA, Hooper-Miele CC, Gilman RH, Steinhoff MC, Ellington LE, Gross M, Price C, Tielsch JM, Checkley W. 2015. Lung ultrasound for the diagnosis of pneumonia in children: A meta-analysis. Pediatrics. 135(4):714-722.

12. Caiulo VA, Gargani L, Caiulo S, Fisicaro A, Moramarco F, Latini G, Picano E. 2011. Lung ultrasound in bronchiolitis: Comparison with chest X-ray. European journal of pediatrics. 170(11):1427.

13. Cattarossi L, Copetti R, Brusa G, Pintaldi S. 2016. Lung ultrasound diagnostic accuracy in neonatal pneumothorax. Canadian respiratory journal. 2016.

14. Martelius L, Heldt H, Lauerma K. 2016. B-lines on pediatric lung sonography: Comparison with computed tomography. Journal of Ultrasound in Medicine. 35(1):153-157.

15. Loi B, Vigo G, Baraldi E, Raimondi F, Carnielli VP, Mosca F, De Luca D. 2021. Lung ultrasound to monitor extremely preterm infants and predict bronchopulmonary dysplasia a multicenter longitudinal cohort study. American Journal of Respiratory and Critical Care Medicine. 203(11):1398-1409.

16. Chiesa AM, Ciccarese F, Gardelli G, Regina UM, Feletti F, Bacchi Reggiani ML, Zompatori M. 2015. Sonography of the normal lung: Comparison between young and elderly subjects. J Clin Ultrasound. 43(4):230-234.

17. Lougheed MD, Leniere C, Ducharme FM, Licskai C, Dell SD, Rowe BH, FitzGerald M, Leigh R, Watson W, Boulet LP. 2012. Canadian thoracic society 2012 guideline update: Diagnosis and management of asthma in preschoolers, children and adults: Executive summary. Can Respir J. 19(6):e81-88.

18. Tsung JW, Kessler DO, Shah VP. 2012. Prospective application of clinician-performed lung ultrasonography during the 2009 h1n1 influenza a pandemic: Distinguishing viral from bacterial pneumonia. Critical ultrasound journal. 4(1):1-10.

19. Varshney T, Mok E, Shapiro AJ, Li P, Dubrovsky AS. 2016. Point-of-care lung ultrasound in young children with respiratory tract infections and wheeze. Emerg Med J. 33(9):603-610.

20. Dankoff S, Li P, Shapiro AJ, Varshney T, Dubrovsky AS. 2017. Point of care lung ultrasound of children with acute asthma exacerbations in the pediatric ed. Am J Emerg Med. 35(4):615-622.

21. Bloomberg GR. 2010. The exacerbation component of impairment and risk in pediatric asthma. Current Opinion in Allergy and Clinical Immunology. 10(2):155-160.

22. Cooper BG, Stocks J, Hall GL, Culver B, Steenbruggen I, Carter KW, Thompson BR, Graham BL, Miller MR, Ruppel G et al. 2017. The global lung function initiative (gli) network: Bringing the world's respiratory reference values together. Breathe (Sheff). 13(3):e56-e64.

23. Becker A, Berube D, Chad Z, Dolovich M, Ducharme F, D'Urzo T, Ernst P, Ferguson A, Gillespie C, Kapur S et al. 2005. Canadian pediatric asthma consensus guidelines, 2003 (updated to december 2004): Introduction. Cmaj. 173(6 Suppl):S12-14.

24. Copetti R, Cattarossi L. 2008. Ultrasound diagnosis of pneumonia in children. Radiol Med. 113(2):190198.

25. Volpicelli G, Elbarbary M, Blaivas M, Lichtenstein DA, Mathis G, Kirkpatrick AW, Melniker L, Gargani L, Noble VE, Via G et al. 2012. International evidence-based recommendations for point-of-care lung ultrasound. Intensive Care Med. 38(4):577-591.

26. Juniper EF, Gruffydd-Jones K, Ward S, Svensson K. 2010. Asthma control questionnaire in children: Validation, measurement properties, interpretation. Eur Respir J. 36(6):1410-1416.

27. Lai CK, Beasley R, Crane J, Foliaki S, Shah J, Weiland S. 2009. Global variation in the prevalence and severity of asthma symptoms: Phase three of the international study of asthma and allergies in childhood (isaac). Thorax. 64(6):476-483. 
28. Anderson H, Gupta R, Kapetanakis V, Asher M, Clayton T, Robertson C, Strachan D. 2008. International correlations between indicators of prevalence, hospital admissions and mortality for asthma in children. International Journal of Epidemiology. 37(3):573-582.

29. Basile V, Di Mauro A, Scalini E, Comes P, Lofu I, Mostert M, Tafuri S, Manzionna MM. 2015. Lung ultrasound: A useful tool in diagnosis and management of bronchiolitis. BMC pediatrics. 15(1):1-8.

30. Shah VP, Tunik MG, Tsung JW. 2013. Prospective evaluation of point-of-care ultrasonography for the diagnosis of pneumonia in children and young adults. JAMA Pediatrics. 167(2):119.

31. Eggleston PA, Ward BH, Pierson WE, Bierman CW. 1974. Radiographic abnormalities in acute asthma in children. Pediatrics. 54(4):442-449.

32. Acosta CM, Maidana GA, Jacovitti D, Belaunzaran A, Cereceda S, Rae E, Molina A, Gonorazky S, Bohm SH, Tusman G. 2014. Accuracy of transthoracic lung ultrasound for diagnosing anesthesia-induced atelectasis in children. Anesthesiology. 120(6):1370-1379.

33. Park JW, Hong YK, Kim CW, Kim DK, Choe KO, Hong CS. 1997. High-resolution computed tomography in patients with bronchial asthma: Correlation with clinical features, pulmonary functions and bronchial hyperresponsiveness. Journal of investigational allergology \& clinical immunology. 7(3):186-192.

34. Paganin F, Trussard V, Seneterre E, Chanez P, Giron J, Godard P, Senac J, Michel F, Bousquet J. 1992. Chest radiography and high resolution computed tomography of the lungs in asthma. American Review of Respiratory Disease. 146(4):1084-1087.

\section{Hosted file}

Table LUS in pediatric asthma.docx available at https://authorea.com/users/451387/articles/ 549568-lung-ultrasound-in-pediatric-asthma-characterizing-baseline-findings-outside-ofacute-exacerbations 


\section{6 eligible}

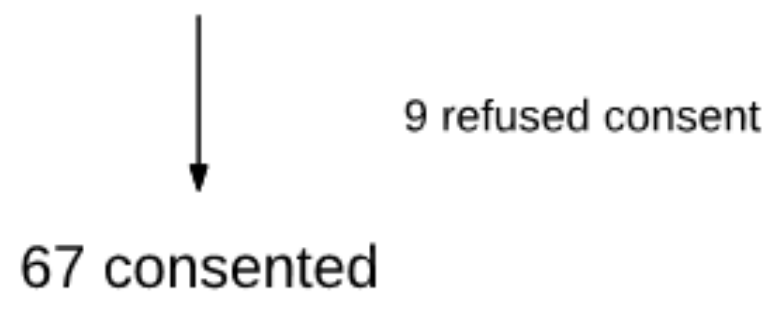

8 excluded due to recent infection

59 enrolled

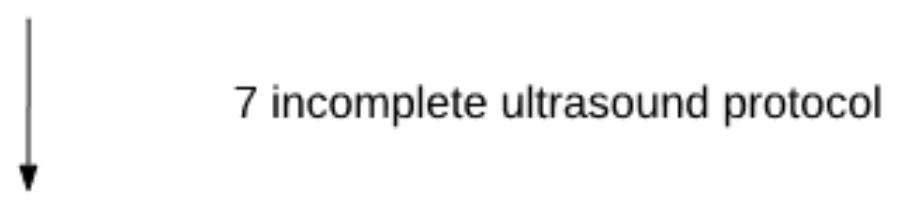

52 included in analysis

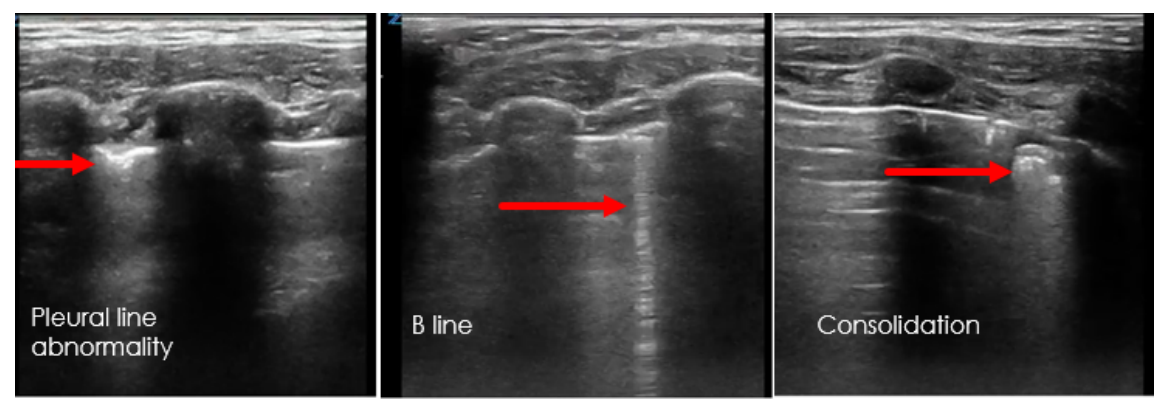




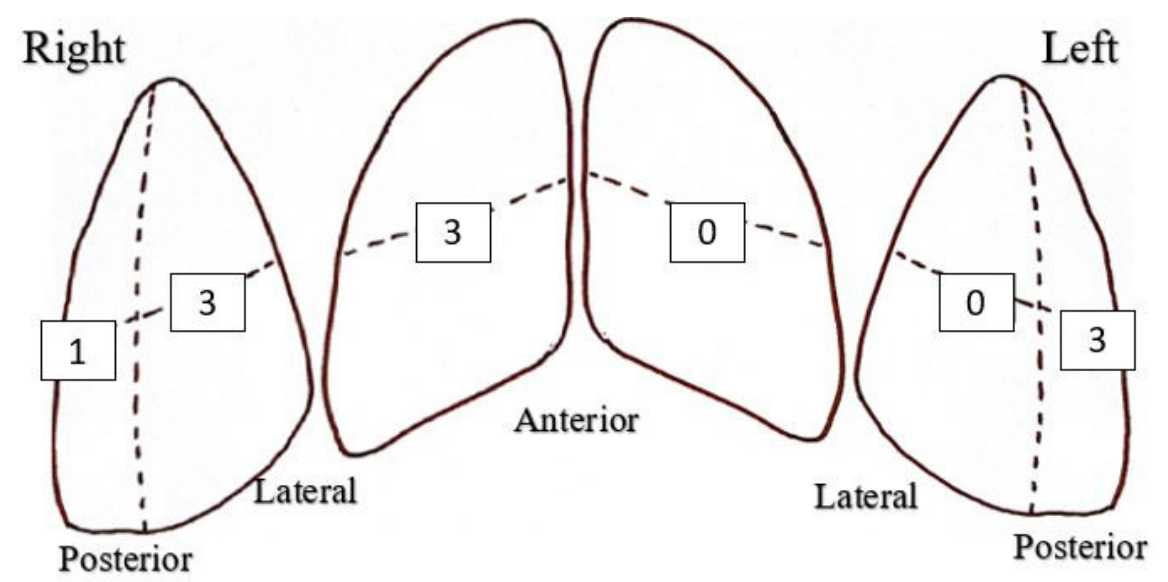

